OPEN ACCESS

Edited by:

Jun Deng,

Shanghai Cancer Institute, China

Reviewed by:

Vladimir Tesar,

Charles University, Czechia

Anna Larissa Janes,

Federal University of São Paulo, Brazil

${ }^{*}$ Correspondence:

Dragana Odobasic

dragana.odobasic@monash.edu

Specialty section:

This article was submitted to

Autoimmune and

Autoinflammatory Disorders,

a section of the journal

Frontiers in Immunology

Received: 15 December 2020 Accepted: 08 July 2021 Published: 29 July 2021

Citation:

Odobasic D and Holdsworth SR (2021) Emerging Cellular Therapies for Anti-myeloperoxidase Vasculitis and

Other Autoimmune Diseases.

Front. Immunol. 12:642127. doi: 10.3389/fimmu.2021.642127

\section{Emerging Cellular Therapies for Anti-myeloperoxidase Vasculitis and Other Autoimmune Diseases}

\author{
Dragana Odobasic ${ }^{1 *}$ and Stephen R. Holdsworth ${ }^{1,2,3}$ \\ ${ }^{1}$ Centre for Inflammatory Diseases, Department of Medicine, Monash University, Monash Medical Centre, Clayton, \\ VIC, Australia, ${ }^{2}$ Department of Nephrology, Monash Health, Clayton, VIC, Australia, ${ }^{3}$ Department of Immunology, Monash \\ Health, Clayton, VIC, Australia
}

Anti-myeloperoxidase vasculitis (MPO-AAV) is a life-threatening autoimmune disease which causes severe inflammation of small blood vessels, mainly in the kidney. As for many other autoimmune diseases, current treatments, which consist of general immunosuppressants, are partially effective, toxic and broadly immunosuppressive, causing significant and serious adverse effects in many patients. Therefore, there is an urgent need for more targeted and less harmful therapies. Tolerogenic dendritic cells, regulatory $T$ cells and stem cells have emerged as attractive, new and safer options for the treatment for various autoimmune diseases due to their unique and selective immunosuppressive capacity. In this review, we will discuss how these cellular therapies offer potential to become novel and safer treatments for MPO-AAV.

Keywords: vasculitis, glomerulonephritis, myeloperoxidase, tolerogenic dendritic cells, regulatory $\mathbf{T}$ cells, stem cells

\section{INTRODUCTION}

Anti-neutrophil cytoplasmic antibody (ANCA)-associated vasculitis (AAV) is a severe condition which causes inflammation and damage of small blood vessels. It is caused by autoimmunity against neutrophil proteins, mainly myeloperoxidase (MPO) and proteinase-3 (PR3). AAV consists of microscopic polyangiitis (MPA), granulomatosis with polyangiitis (GPA) and eosinophilic granulomatosis with polyangiitis (EGPA), and it has an annual incidence of 20/million $(1,2)$. MPO-ANCA are found in the majority of MPA and a smaller proportion of GPA and EGPA patients, while PR3-ANCA are found predominantly in GPA (3).

Although MPO-AAV and PR3-AAV have many similarities, they are now considered to be different diseases based on their epidemiology, genetics, etiology, immunopathology and clinical features. MPO-AAV predominates in southern Europe and Asia-Pacific, is genetically weakly associated with HLA-DQ, mainly occurs as a single event, and vasculitis is mostly limited to the kidney $(1,4)$. In contrast, PR3-AAV is more commonly found in the northern hemisphere, is associated with HLA-DP, has a higher rate of relapse, and organs other than the kidneys such as the lungs are also affected $(1,4)$.

As in many other human autoimmune diseases, the aim of conventional therapies in AAV has been to damage the immune system in general to attenuate the autoimmunity-induced inflammatory injury to organs expressing the target autoantigens. The development of biological 
therapies, mainly monoclonal antibodies, has allowed more accurate selection and targeting of key components of pathways which mediate auto immunopathogenesis. These are more "precise" than conventional therapies in having fewer off target injurious effects on the immune system.

However, the ultimate goal for many autoimmune diseases, including $\mathrm{AAV}$, is to restore tolerance (unresponsiveness) towards the disease-causing autoantigen in a way that would turn off only the injurious autoimmune response, without adversely affecting host immune defense and causing other major side effects. Although we now have tools which can restore tolerance in an antigen-specific manner, antigenspecific immunosuppression causing disease reversal is nearly impossible to achieve in many human autoimmune diseases, but is likely to be realistic in AAV, particularly MPO-AAV, due to the reasons explained below. For a biological treatment strategy to deliver successful antigen-specific restoration of tolerance, there are several essential components:

i. The diagnosis of a particular disease must be possible before the immune injury has induced irreversible damage to the target organ. In many human autoimmune diseases such as autoimmune thyroiditis and type 1 diabetes (T1D), this is not possible.

ii. The major human disease-causing autoantigen needs to be known. This is not the case in many diseases including systemic lupus erythematosus (SLE), rheumatoid arthritis (RA) and type 1 diabetes (T1D).

iii. In diseases such as Multiple Sclerosis (MS), epitope spreading makes antigen-specific inhibition complex and difficult to achieve.

iv. There must be thorough and precise knowledge of the key pathways and the essential components of the immune pathogenesis of the disease.

v. Animal models with similar autoimmune target, immune pathogenic pathways and clinical outcomes need to exist or can be developed so that pre-clinical studies can be used to provide proof-of-concept that safe precise biological therapies can be taken on to clinical trials.

MPO-AAV is an autoimmune disease where all these criteria are met. Although patients often present with significant target organ (kidney) damage, at the time of diagnosis, the majority have sufficient kidney reserve to live a long life without the need for transplantation or dialysis once effective therapy is introduced. The major disease-causing autoantigen (MPO) is known, there is no evidence of epitope spreading occurring, and relevant animal models have been developed that have helped define the immunopathogenesis of the human disease and identify new therapeutic targets. On the other hand, although humanized mice have emerged as a promising tool to develop successful in vivo models of PR3-AAV (5), murine models of this disease have been difficult to induce (6). This is most likely because PR3 is not detected on mouse neutrophils and is therefore not accessible to anti-PR3 antibodies (1). Hence, this review will focus mainly on MPO-AAV. However, the potential cellular therapies discussed below may be also applied to PR3AAV and later tested in relevant animal models.

Autologous ex vivo-generated regulatory $\mathrm{T}$ cells (Tregs) and autoantigen-loaded tolerogenic dendritic cells (DCs) are an appealing tool for the treatment of autoimmune diseases, including MPO-AAV, because they can provide safe, antigen-specific immunosuppression, without posing any risk of rejection. Alternative to inducing MPO-specific immunosuppression, other attractive cell therapies such as stem cells would desirably suppress anti-MPO autoimmunity and vasculitis without being rejected or producing major adverse effects. This review will discuss emerging evidence which suggests that such cellular therapies may offer a safer, effective therapeutic option for MPO-AAV. Figure 1 illustrates how these therapies could be used in this disease, with their main advantages and disadvantages summarized in Table $\mathbf{1}$.

\section{MPO-AAV}

MPO-AAV is caused by autoimmunity to MPO, an abundant protein found inside our most common immune cell, neutrophil $(3,7)$. This disease causes severe inflammation and destruction of small blood vessels, leading to significant morbidity and mortality. It equally affects men and women, mainly over the age of 50, but it can also affect young adults and children (1). MPO-AAV is associated with the presence of serum anti-MPO antibodies, known as anti-neutrophil cytoplasmic antibodies or MPO-ANCA. Although the kidney bares the brunt of autoimmune injury, the major autoantigen, MPO, is not normally expressed in the kidney. MPO is, however, the major protein present in the granules of neutrophils (8). Evidence from relevant human and mouse studies shows that circulating MPOANCA target activated, MPO-exposing neutrophils, which subsequently lodge in glomeruli and deposit the autoantigen there (9-16). It also shows that both MPO-ANCA and MPOspecific CD4 $\mathrm{T}$ cells vitally contribute to the development of glomerular injury in MPO-AAV (9-16).

Animal models have provided a great deal of knowledge about the immunobiology of MPO-AAV. A few different animal models of MPO-AAV exist, including an antibody transfer mouse model in which injury is mediated by passively-transferred MPO-ANCA, transplanting irradiated, MPO-immunized MPO-deficient mice with wildtype bone marrow and immunizing WKY rats with MPO. They all closely resemble human disease, immunologically and pathologically, and have been thoroughly reviewed elsewhere (17). In one model, wildtype mice are immunized with MPO to induce active MPO-specific autoimmunity, including MPOANCA and MPO-specific T cells $(16,18-21)$. They are then administered either MPO-ANCA or low dose anti-glomerular basement membrane (GBM) globulin which cause neutrophils to deposit MPO in glomeruli for subsequent recognition by infiltrating MPO-specific T cells. In line with data from MPOAAV patients, CD4 $(13,22)$ and CD8 T cells $(22,23)$, neutrophils 

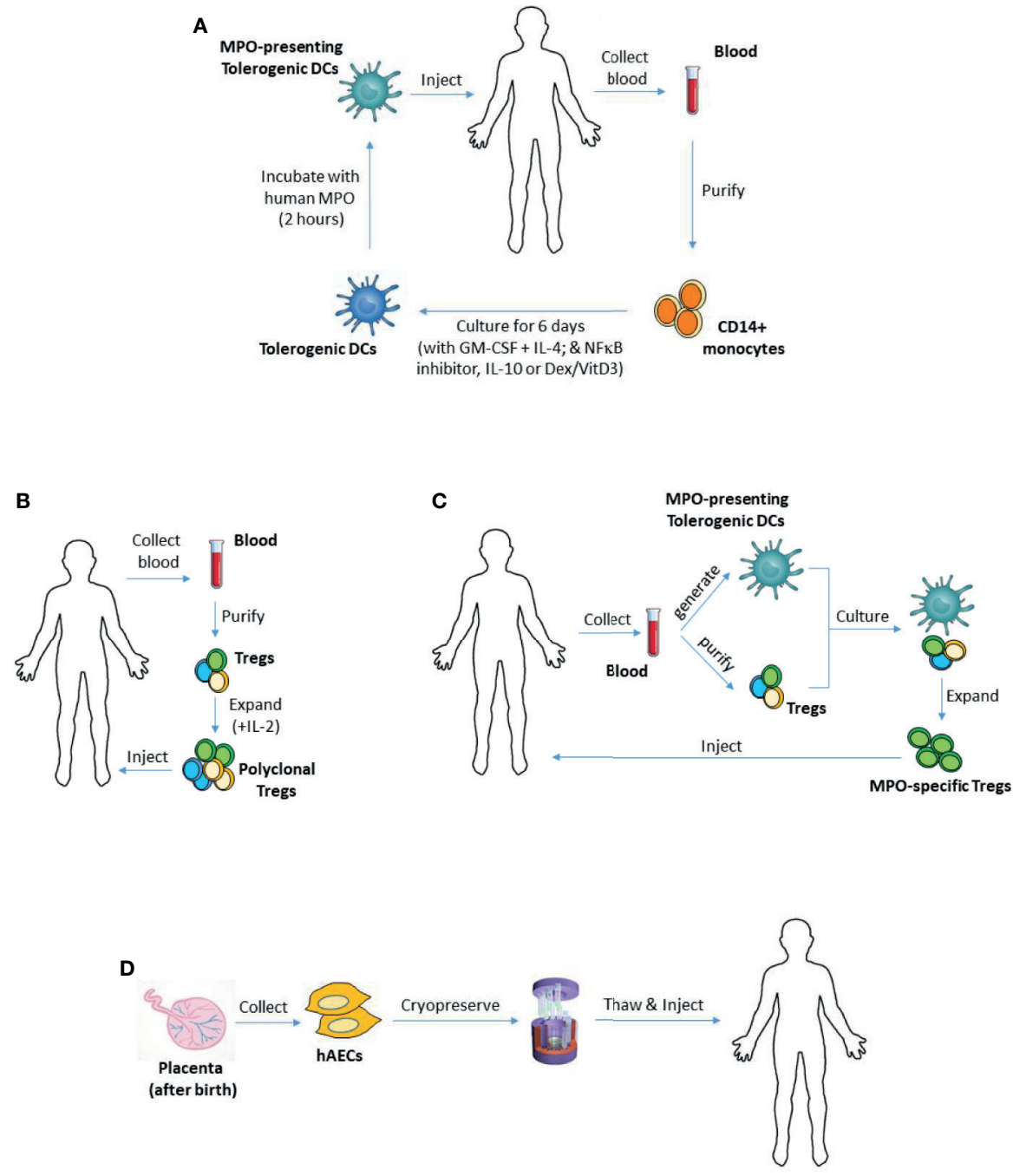

FIGURE 1 | Using tolerogenic DCs, Tregs or hAECs as cellular therapies in MPO-AAV. (A) To generate monocyte-derived DCs, CD14+ monocytes can be purified from patient's blood and cultured in the presence of GM-CSF and IL-4. Different types of tolerogenic DCs can be generated by adding various anti-inflammatory mediators to the culture including IL-10, DexNitD3 or inhibitors of NFkB. To make the DCs present MPO, they are then pulsed with purified human MPO, and injected back into the patient. (B) To expand polyclonal Tregs ex vivo, patient's Tregs $\left(C D 4^{+} C D 25^{+} C D 127^{-1 / o m}\right)$ can be isolated from their blood, then cultured for several weeks in the presence of IL-2. (C) Patient's MPO-presenting tolerogenic DCs and Tregs, generated and purified as in (A, B) respectively, could be cocultured to expand MPO-specific Tregs. Such antigen-specific Tregs are expected to have superior suppressive capacity compared with polyclonal Tregs. (D) hAECs are isolated from the amniotic membrane of the placenta after birth and cryopreserved as primary (non-cultured) cells. They can be given to MPO-AAV patients when needed. DCs, dendritic cells; Tregs, regulatory T cells; hAECs, human amniotic epithelial cells; MPO, myeloperoxidase; AAV, ANCA-associated vasculitis; GM-CSF, granulocyte-macrophage colony-stimulating factor; IL-4, interleukin 4; Dex, dexamethasone; VitD3, vitamin D3; NFkB, nuclear factor kappa B.

and macrophages $(13,22,24), \operatorname{IL}-17 \mathrm{~A}(19,25)$ and $\operatorname{IFN} \gamma(26,27)$ promote injury in this model, while $\mathrm{CD} 4+$ foxp $3+$ regulatory $\mathrm{T}$ cells (Tregs) are inhibitory (28-30). Here, our group has also defined the disease-causing immunodominant MPO T cell peptide (16), which is strikingly similar to the immunodominant human MPO peptide $(16,20)$. Hence, studies of MPO-specific immunomodulation in this model are very relevant to human disease.

As for many other autoimmune diseases, current treatments for MPO-AAV are only partially effective, but are harmful and non-specific, thus causing significant serious side effects in many patients which lead to considerable complications and death. Decades after their introduction, the first-line therapy for induction of remission still consists of high-dose corticosteroids and cyclophosphamide (1). These treatments induce remission in $70-90 \%$ of patients, but the incidence of dialysis or death at 5 years is still high $(30 \%)(3,31,32) .1$ in 3 patients also relapse while being treated $(1,31)$. The main problem with these therapies is that they are highly toxic and broadly immunosuppressive. Cyclophosphamide increases the risk of infection, cancer and infertility, while corticosteroids cause cardiovascular problems, diabetes, depression, anxiety, 
TABLE 1 | Advantages and disadvantages of different cellular therapies for human MPO-AAV.

\begin{tabular}{|c|c|c|}
\hline Cellular therapy & Advantages & Disadvantages \\
\hline \multirow{4}{*}{$\begin{array}{l}\text { Antigen-loaded } \\
\text { tolerogenic DCs }\end{array}$} & - antigen-specific & - cost related to cell isolation and in-vitro culture \\
\hline & - no risk of rejection (using patients' own ex vivo-modified cells) & - long-term stability and survival after transfer needs to be optimized \\
\hline & - abundant DCs can be generated from patients' PBMCs & - migratory capacity can be impaired with certain tolerogenic treatments \\
\hline & - expensive genetic engineering methods not required & \\
\hline \multirow[t]{4}{*}{ Polyclonal Tregs } & - expensive genetic engineering methods not required & - not antigen-specific \\
\hline & - no risk of rejection (using patients' own ex vivo-expanded & - less suppressive than antigen-specific Tregs \\
\hline & cells) & - reduced stability during in vitro expansion due to the loss of foxp3 \\
\hline & & - cost related to cell isolation and in vitro culture \\
\hline \multirow[t]{5}{*}{ Antigen-specific Tregs } & - antigen-specific & - some approaches use expensive genetic engineering methods (e.g. specific \\
\hline & - no risk of rejection (using patients' own ex vivo-modified cells) & TCR-Tregs and CAR-Tregs) \\
\hline & - more suppressive than polyclonal Tregs & $\begin{array}{l}\text { - difficulty to generate specific TCR-Tregs due to the immunodominant } \\
\text { peptide of human MPO being unknown }\end{array}$ \\
\hline & & $\begin{array}{l}\text { - CAR-Tregs may become systemically hyper-activated due to widespread } \\
\text { expression of MPO }\end{array}$ \\
\hline & & - cost related to cell isolation and in vitro culture \\
\hline \multirow[t]{6}{*}{ hAECS } & - low immunogenicity and risk of rejection & - not antigen-specific, thus pose a risk of more broadly suppressing immunity \\
\hline & - low risk of causing tumors & - low long-term survival after administration \\
\hline & $\begin{array}{l}\text { - offer protection against infection, cancer and cardiovascular } \\
\text { disease }\end{array}$ & \\
\hline & - unique immunosuppressive capacity & \\
\hline & $\begin{array}{l}\text { - isolation from the amnion after birth is non-invasive, easy, fast, } \\
\text { relatively cheap and ethical }\end{array}$ & \\
\hline & - plentiful cells are isolated from an abundant source (placenta) & \\
\hline
\end{tabular}

insomnia, bone loss and gastric ulcers, as well as an increased risk of infection $(32,33)$. The rate of infection can be potentially reduced by lowering cumulative doses of corticosteroids or replacing them with avacopan (complement C5a receptor inhibitor) $(34,35)$. Rituximab (B cell-depleting monoclonal antibody) has been approved for use instead of cyclophosphamide, but it induces similar rates of infections, mainly due to reduced numbers of $\mathrm{B}$ cells, hypogammaglobulinemia and late-onset neutropenia $(32,36)$. Of all patient deaths in AAV, an alarming $70 \%$ are caused by treatment-related side effects, mostly infections (31). Another common downside of these therapies, including past and currently-ongoing clinical trials in ANCA vasculitis (1), is that none of them specifically target anti-MPO autoimmunity to avoid complications due to their off-target, non-antigen-specific effects. There is an urgent need for safer, more-targeted effective therapies.

\section{TOLEROGENIC DCs}

DCs are specialized immune cells and most-potent antigenpresenting cells which vitally control adaptive immunity. After activation, they upregulate MHC-II, costimulatory molecules (e.g. CD40, CD80/86) and pro-inflammatory cytokines (e.g. IL-12, TNF). In this context, they present antigen to $\mathrm{T}$ cells via MHC-II to induce protective antigen-specific $\mathrm{T}$ cell immunity against pathogens. In contrast, DCs are also critical to the maintenance of peripheral tolerance by presenting self-antigens in an immature or semi-mature state, thus causing $\mathrm{T}$ cell hyporesponsiveness, as shown by studies in which DC depletion during steady-state resulted in fatal autoimmunity $(37,38)$. Tolerogenic DCs are found throughout the body including mucosal surfaces where they promote airway and oral tolerance and unresponsiveness towards commensal microbiota (37). They generally express high levels of antiinflammatory (e.g. PD-L1, IL-10, TGF $\beta$ ) and low levels of proinflammatory mediators (e.g. CD40, CD80/86, IL-12) (37).

Tolerogenic DCs can be also made ex vivo by modification with various anti-inflammatory agents including IL-10, Dexamethasone (Dex; glucocorticoid), VitaminD3 (VitD), inhibitors of NFKB (one of their major pro-inflammatory pathways) and tools such as antisense oligonucleotides (oligos) which can inhibit gene expression of molecules critical for T cell activation like CD80, CD86 and CD40 (39-46). These DCs use various molecules to inhibit pathogenic T cells (e.g. PD-L1, IL-10, TGF $\beta$ ), and can turn them off by promoting apoptosis, and by inducing endogenous inhibitory cells such as CD4+foxp3+ Tregs, type 1 regulatory cells (Tr1; inhibitory CD4+foxp3-) and regulatory B cells (Bregs) $(39,40,47)$. However, mechanisms of immunosuppression are context and disease-dependent and vary between differentlymodified DCs which exhibit different phenotypes.

Various antigen-loaded tolerogenic DCs, including CD40deficient DCs, DCs treated with Dexamethasone (Dex)/Vitamin $\mathrm{D}$ (VitD) or NFKB inhibitors (e.g. BAY-11-7082), have rapidly, in 2-3 days, induced potent antigen-specific immunosuppression and attenuated organ damage in models of allergy, transplantation and autoimmune diseases (e.g. RA, MS, T1D), while generally remaining very stable after transfer (41, 48-51). These DCs have induced long-lasting immunosuppression, although they themselves have persisted for only a few weeks in recipients (52). Due to their stability and capacity to provide antigen-specific immunosuppression, as shown in rodents and non-human primates $(41,48-51)$, autologous ex vivo-derived 
tolerogenic DCs have emerged as an excellent therapeutic candidate for the treatment of autoimmune diseases. Their clinical use in autoimmunity is now a reality, with 5 phase I trials completed in RA, T1D, MS, neuromyelitis optica (NMO) and Crohn's disease (53-57). In these clinical studies, patients' own ex vivo-modified tolerogenic DCs (including BAY, Dex/VitD and CD40/80/86 anti-sense oligo-treated DCs), which were biologically active, were shown to be safe and well-tolerated, without producing any major side effects for 12 months. Furthermore, it was shown that tolerogenic DC therapy produced anti-inflammatory and immunomodulatory effects in these trials, with some positive clinical outcomes. For example, in RA, the DCs decreased effector $\mathrm{T}$ cells and their ability to produce IL-6, increased the ratio of Tregs: effector $\mathrm{T}$ cells, reduced serum levels of pro-inflammatory cytokines and chemokines and diminished the disease activity score (53). In T1D, tolerogenic DCs increased the frequency of peripheral Bregs (54), while in MS/NMO, they upregulated IL-10 production by PBMCs and reduced memory CD8 $\mathrm{T}$ cells (57). In Crohn's disease, tolerogenic DCs reduced the disease activity index, with 1 patient reaching clinical remission and 2 a positive clinical response, and disease lesions markedly improving in 3 out of 9 patients (55). These studies demonstrate the feasibility and safety of this therapy in humans, with promising clinical outcomes which need to be further investigated in future trials. The optimum and most effective treatment regimen still needs to be determined for each autoimmune condition including DC dose, route and frequency of administration, long-term stability and survival, and the type of tolerogenic DC to be used. It also needs to be ensured that these cells can migrate to lymphoid organs to be able to exert their effects since many antiinflammatory agents used to make tolerogenic DCs can inhibit their migratory capacity.

Recently, we demonstrated that administration of ex vivogenerated antigen-presenting tolerogenic DCs can induce selective, MPO-specific immunosuppression and attenuate vasculitis in mice (58). Bone marrow-derived tolerogenic DCs were generated by treatment with an NFKB inhibitor (BAY-117082) and pulsed with mouse MPO. These MPO-presenting inhibitory DCs were then given to mice with established antiMPO autoimmunity. The DCs significantly decreased vasculitis and MPO-specific immunity, including effector CD4 $\mathrm{T}$ cell activation, proliferation, survival and pro-inflammatory cytokine production, as well as CD8 T cell and B cell responses. In line with suppressing anti-MPO immunity, MPO/BAY DCs upregulated Treg expression of inhibitory mediators including foxp3, CTLA-4, TNFR2 and IL-10, without affecting $\operatorname{Tr} 1$ or Bregs. Studies in Treg-depleted mice showed that the inhibitory effects of MPO/BAY DCs on antiMPO autoimmunity were dependent on Tregs. Subsequent adoptive transfer/antibody blockade experiments showed that MPO/BAY DC-induced Tregs suppressed anti-MPO immunity and vasculitis via IL-10. Further in vitro DC : Treg co-culture experiments, supported by in vivo antibody blockade studies, showed that MPO/BAY DCs induced IL-10+ Tregs via the ICOS/ICOS-ligand pathway.
Importantly, the above-described inhibitory effects of MPO/ BAY DCs on anti-MPO immunity were MPO-specific, since the same DCs did not induce Tregs or suppress immunity against an irrelevant antigen. However, in line with augmenting Th2 responses, MPO/BAY DCs did increase circulating IgE levels in recipient mice, indicating that they may adversely exacerbate allergy, but further studies are needed to test that assertion.

Overall, these pre-clinical, proof-of-concept studies demonstrated that MPO-presenting tolerogenic DCs may be a potential MPO-specific therapy for MPO-AAV which deserve further exploration. Future studies will be needed to test other types of MPO-pulsed tolerogenic DCs in pre-clinical models of MPO-AAV because the effectiveness and precise mechanism of immunosuppression varies between different types of tolerogenic DCs which exhibit different phenotypes. This will allow the best DC candidate to be identified for further studies and progression to clinical trials in MPO-AAV. In addition, before such a cellular therapy could be clinically tested, it would be important to determine if patients' own MPO-presenting tolerogenic DCs could selectively turn off their anti-MPO T cell responses ex vivo.

\section{TREGS}

Tregs are a specialized inhibitory subset of CD4+ T cells characterized by expression of $\mathrm{CD} 25$ and foxp3, a transcription factor essential for their development, stability and suppressive capacity (59). In humans, including MPO-AAV patients, they are also CD127-low $(60,61)$. Tregs play a vital role as regulators of pathogenic immunity in various immunemediated conditions such as allergy, transplantation and inflammation. They are well known to critically maintain peripheral tolerance by inhibiting pathogenic autoreactive $\mathrm{T}$ cells and enhancing the tolerogenic capacity of DCs in an antigen-specific manner $(62,63)$. Tregs can also inhibit immunity and subsequent inflammation leading to organ damage by suppressing other types of injurious immune cells such as neutrophils, macrophages and B cells (62). They provide immunosuppression by expressing various inhibitory mediators including IL-10, TGF $\beta$, IL-35, CTLA-4 and TNFR2 (58, 64-66).

Dysregulation of Treg number and/or function has been associated with the development of several autoimmune diseases including systemic lupus erythematosus, RA, T1D and MS. Similarly, several studies have shown that the frequency and suppressive capacity of Tregs is significantly reduced and negatively correlates with disease in MPO-AAV patients (28, 30). The critical importance of endogenous Tregs as negative regulators of anti-MPO autoimmunity and vasculitis has been confirmed in experimental MPO-AAV. We have shown that Tregs not only inhibit the generation of anti-MPO autoimmunity (29), but that they also suppress established responses of MPO-specific CD4 $\mathrm{T}$ cells, CD8 $\mathrm{T}$ cells and $\mathrm{B}$ cells (58).

Due to their ability to provide antigen-specific immunosuppression, there has been a great deal of interest to 
develop and use autologous ex-vivo-derived Tregs as a potential therapy for autoimmune diseases. Several studies have shown that such Tregs can attenuate pathogenic immunity and thus organ damage in models of transplantation and autoimmunity (67-69). Phase I trials in autoimmune conditions have demonstrated their feasibility and safety in humans $(70,71)$. However, many optimizations are still required before Tregs can be clinically used such as enhancing their stability and survival post transfer and determining optimum dose and frequency of administration.

Ex vivo-derived Tregs, which have been tested as cellular therapies in animal models and human trials, can be broadly categorized as either polyclonal or antigen-specific Tregs.

\section{Polyclonal Tregs}

Many protocols expand Tregs for several weeks with Treg growth factors such as IL-2, without antigen-stimulation (64). This generates polyclonal Tregs of broad specificities. Such Tregs have attenuated immune-mediated organ damage in models of autoimmune conditions and shown relative safety and some efficacy in human trials (e.g. T1D) $(70,71)$. However, polyclonal Tregs are much less suppressive than their antigen-specific counterparts (72) and they become less stable and suppressive even before administration due to their progressive loss of foxp3 expression during in vitro expansion (73). Therefore, although not ideal, autologous ex vivo-expanded polyclonal Tregs could be trialled as a treatment in MPO-AAV patients, but their therapeutic efficacy needs to be first tested in pre-clinical models of the disease.

\section{Antigen-Specific Tregs}

Antigen-specific Tregs are a lot more attractive and promising due to their specificity and superior suppressive capacity. They can be generated in different ways to produce Tregs with an antigen-specific $\mathrm{T}$ cell receptor (TCR), chimeric antigen receptor (CAR) Tregs or DC-induced Tregs.

\section{Antigen-Specific TCR Tregs}

Tregs can be transduced in order to express a high-affinity TCR specific for the autoantigen of interest. Such geneticallyengineered Tregs have been effective in models of autoimmune diseases such as T1D (68). Some of the biggest challenges in generating autoantigen-specific TCR Tregs for the treatment of autoimmunity have been the lack of knowledge of the diseasecausing autoantigen and its immunodominant epitope(s) in most conditions including SLE and RA, as well as antigen and epitope shifting in others such as T1D and MS.

In MPO-AAV, the autoantigen (MPO) is known. Our group has also defined the $\mathrm{T}$ cell specific MPO immunodominant epitope in a murine model of the disease, which interestingly shows striking homology with the human MPO dominant peptide recognized by patients' pathogenic MPO-ANCA (16, 20). Hence, it may be possible to generate murine MPO-specific TCR and test them in pre-clinical models of MPO-AAV in the near future. The human T cell-specific MPO dominant epitope has not yet been identified, however recent studies suggest that there may be several such epitopes present within human MPO
(74), thus making the generation of human MPO-specific TCR Tregs for the treatment of MPO-AAV patients more challenging.

\section{CAR Tregs}

There has also been a great deal of interest in developing CAR Tregs for the treatment of autoimmune conditions. CAR Tregs are genetically-engineered Tregs which contain an extracellular CAR molecule (autoantigen-binding antibody domain), a transmembrane region and an intracellular $\mathrm{T}$ cell signaling domain. CAR Tregs are able to migrate to the site of auto-inflammation where they get activated by their specific autoantigen (69). However, for this therapy to be effective, the autoantigen needs to be expressed only at the diseased site. If the autoantigen is also expressed elsewhere in the body, this could cause systemic over-activation of the Tregs, potentially leading to side effects associated with broader immunosuppression. The potential therapeutic efficacy and safety of CAR Tregs in MPO-AAV may be questionable. This is mainly due to the fact that MPO is not only expressed in the target organ (kidney), but that neutrophils, the major source of MPO, and to a lesser extent monocytes/macrophages, also release it into the extracellular space following cell activation in response to bacteria and other pathogens. MPO is also released from neutrophil precursors during its synthesis in the bonemarrow, so this autoantigen is always present in the circulation $(8,75)$.

\section{DC-Induced Tregs}

Antigen-specific Tregs can be expanded ex vivo from the polyclonal repertoire without genetic engineering by using antigen-presenting DCs. This approach has been largely employed in transplantation due to the knowledge of alloantigens $(76,77)$. However, it could be also applied in autoimmune conditions in which the autoantigen is known, including MPO-AAV. For example, ex vivo DC-induced/ expanded Tregs were effective at reducing immunity and organ damage in a model of RA (78). DC-induced Tregs have increased suppressive capacity and stability due to the long-lasting, enhancing effects of DCs on Treg foxp3 expression and stability $(76,78)$.

We have recently shown that Tregs induced by MPOpresenting tolerogenic DCs can be used to inhibit established anti-MPO immunity and vasculitis in a pre-clinical model of MPO-AAV (58). In these studies, administration of DC-induced CD4+foxp3+ Tregs suppressed MPO-specific autoimmunity including CD4 T cells, CD8 T cells and B cells, and attenuated vasculitis. These proof-of-concept studies suggested that MPOspecific Tregs induced/expanded by MPO-presenting DCs may be a potential and feasible therapy for MPO-AAV. However, the long-term stability and effect of such Tregs needs to be explored in further studies.

Therefore, although many challenges still remain and the therapeutic efficacy of various types of Tregs needs to be tested in pre-clinical models of MPO-AAV, cell therapy utilizing patients' own ex vivo-modified Tregs, particularly antigen-specific ones, remains a promising potential treatment option for vasculitis patients worth further exploring. 


\section{STEM CELLS}

Stem cells have also emerged as a promising therapeutic approach for the treatment of various inflammatory and autoimmune diseases due to their immunomodulatory ability. Several types of stem cells, including embryonic and mesenchymal stem cells (MSC), have attenuated organ damage in models of immune-mediated diseases and their safety and efficacy have been evaluated in clinical trials (79). One particular stem cell type, human amniotic epithelial cells (hAECs), have gained much attention in recent years as a treatment choice due to their safety and clinical applicability.

hAECs, which have pluripotent stem cell properties $(80,81)$, represent a novel, safe and affordable therapeutic option for MPO-AAV, for several reasons. They are isolated after birth from the amniotic which is attached to the placenta (82), therefore bypassing ethical barriers that normally occur with other (e.g. embryonic) stem cells. hAEC isolation involves noninvasive, easy, fast and low-cost procedures, resulting in an abundance of readily-available cells which are infused as primary, non-passaged cells (82). In contrast, other types of stem cells (e.g. mesenchymal and embryonic) have ethical issues regarding their isolation or have to be cultured for several weeks to generate enough infusible cells, thus significantly increasing the cost and potential for in vitro mal-transformation. hAECs have low immunogenicity because they do not express class IA antigens (HLA-A, HLA-B, HLA-C) or HLA-DR (class II), and are therefore not rejected upon transfer, nor do they form teratomas due to lacking telomerase, as shown in animals and humans $(81,83,84)$.

Importantly, consistent with their role to protect the fetus from mother's immune system, hAECs, like other stem cells, are immunosuppressive. In fact, similar to $\operatorname{MSC}(85,86)$, the protective effects of hAECs are largely due to their paracrine action and immunosuppressive capacity, rather than multilineage differentiation potential. They have suppressed pathogenic immunity by inhibiting effector $\mathrm{T}$ cells, altering macrophage polarisation toward the anti-inflammatory M2 phenotype, inhibiting neutrophils $(87,88)$ or by inducing other immunosuppressive cells such as Tregs and Bregs and as such they have attenuated organ damage in models of various inflammatory and autoimmune diseases including MS, autoimmune thyroiditis and uveitis, lung injury, liver fibrosis (89-94) and stroke (95). Similar inhibitory effects of hAECs on human $\mathrm{T}$ cells have been reported in vitro. For example, in coculture experiments with human PBMCs or purified CD4 T cells, hAECs significantly decreased their proliferation and Th1/Th17 cytokine production $(96,97)$.

hAECs express a range of immunosuppressive mediators, including transforming growth factor beta (TGF $\beta$ ), prostaglandin- $\mathrm{E}_{2}\left(\mathrm{PGE}_{2}\right)$ and the immunosuppressive HLA-G $(81,89)$, which they use to suppress immunity. However, which anti-inflammatory mediators are utilized and which immune cells are targeted by the hAECs depends on the model used. For example, in bleomycin-induced lung injury, hAECs attenuated disease by inducing Tregs via TGF $\beta$ (91). In a model of MS, hAECs suppressed lymphocyte proliferation via TGF $\beta$ and $\mathrm{PGE}_{2}$
(89), but in those studies, hAEC-mediated suppression of disease was not associated with Treg induction. This shows that Tregs are required for hAEC-exerted effects in some, but not all, inflammatory models. Interestingly, but similar to MSC, IFN $\gamma /$ TNF stimulation of hAECs enhances their production of suppressive molecules such as TGF $\beta(86,91)$, suggesting that exposure to pro-inflammatory mediators found in many autoimmune and inflammatory conditions may further augment the inhibitory function of hAECs. Term hAECs are also more suppressive than those from pre-term donors $(<36$ weeks gestation), possibly due to their increased expression of HLA-G (98), which is known to suppress T cell and neutrophil responses and induce Tregs $(99,100)$.

Recently, it was shown that hAECs can also mediate immunosuppression by producing exosomes $(88,101)$. Exosomes are released microvesicles ( $50-100 \mathrm{~nm}$ in diameter) which contain proteins, lipids and DNA/RNA with important roles in intercellular communication (102). They are released by many cell types and have been successfully used as a cell-free therapy in inflammatory conditions $(103,104)$. hAEC-derived exosomes inhibit various immune cells including $\mathrm{T}$ cells, macrophages and neutrophils in vitro and show protection in inflammatory models of acute lung injury and liver fibrosis (88, 101). Therefore, hAEC exosomes may represent a potential cellderived, but cell-free, therapy in MPO-AAV.

Unlike the current AAV treatments, hAECs are unlikely to cause major side effects because they have anti-infection and anti-cancer properties. They produce various anti-microbial mediators including $\beta$-defensins and type I interferons in response to bacteria and viruses in vitro $(105,106)$. They inhibit cancers directly by inducing apoptosis of malignant cells and indirectly by enhancing anti-tumor immunity in vivo $(107,108)$. hAECs also protect against cardiovascular conditions, including stroke and myocardial infarction $(95,109)$.

hAECs have already entered the clinic. Amniotic membrane or hAECs have been safely used to treat eye injuries and promote wound healing for decades $(84,110)$. hAECs are also being tested in clinical trials as a therapy for other conditions. A phase I trial in babies with bronchopulmonary dysplasia has demonstrated their short and long-term feasibility and safety $(111,112)$, while another two phase I trials are underway in liver fibrosis (113) and stroke (114).

Therefore, stem cells such as hAECs represent a potentially safer, effective therapy for MPO-AAV due to their relatively harmless and immunomodulatory profile. Stem cells have never been tested as a treatment in this disease, but we are currently exploring the therapeutic efficacy of hAECs and their exosomes in pre-clinical models of MPO-AAV, which will pave the way for this therapy to be clinically tested in vasculitis patients.

\section{CONCLUSIONS}

Overall, although many obstacles still need to be overcome before tolerogenic cell therapy becomes a reality for vasculitis patients, autologous Tregs and ex vivo-derived MPO-loaded 
tolerogenic DCs offer promise to be a feasible and successful antigen-specific treatment for MPO-AAV. This is because (i) Tregs and tolerogenic DCs have been generally shown to be stable, safe and well-tolerated in patients, and they can uniquely induce antigen-specific immunosuppression in various autoimmune conditions including experimental MPO-AAV, and (ii) MPO-AAV itself does not hold any caveats for effective antigen-specific restoration of tolerance. hAECs, if proven to be effective in pre-clinical models of MPO-AAV, may be even closer to clinical testing in MPO-AAV patients since they have already been extensively characterized, provide an off-the-shelf, abundant and relatively cheap therapeutic option, do not get rejected after transfer and clinical trials in other conditions have demonstrated their safety in humans. If successful, these cell therapies have the potential to change clinical practice in MPO-AAV and provide immense benefit to patients by decreasing their risk of death and complications from

\section{REFERENCES}

1. Hilhorst M, van Paassen P, Tervaert JW, Limburg Renal R. Proteinase 3-ANCA Vasculitis Versus Myeloperoxidase-ANCA Vasculitis. J Am Soc Nephrol (2015) 26(10):2314-27. doi: 10.1681/ASN.2014090903

2. Scott DGI, Watts RA. Epidemiology and Clinical Features of Systemic Vasculitis. Clin Exp Nephrol (2013) 17(5):607-10. doi: 10.1007/s10157013-0830-8

3. Kallenberg CG. Key Advances in the Clinical Approach to ANCAAssociated Vasculitis. Nat Rev Rheumatol (2014) 10(8):484-93. doi: 10.1038/nrrheum.2014.104

4. Lyons PA, Rayner TF, Trivedi S, Holle JU, Watts RA, Jayne DR, et al. Genetically Distinct Subsets Within ANCA-Associated Vasculitis. N Engl J Med (2012) 367(3):214-23. doi: 10.1136/ard.2009.108043

5. Little MA, Al-Ani B, Ren S, Al-Nuaimi H, Leite M Jr, Alpers CE, et al. AntiProteinase 3 Anti-Neutrophil Cytoplasm Autoantibodies Recapitulate Systemic Vasculitis in Mice With a Humanized Immune System. PLoS One (2012) 7(1):e28626. doi: 10.1371/journal.pone.0028626

6. van der Geld YM, Hellmark T, Selga D, Heeringa P, Huitema MG, Limburg PC, et al. Rats and Mice Immunised With Chimeric Human/Mouse Proteinase 3 Produce Autoantibodies to Mouse Pr3 and Rat Granulocytes. Ann Rheum Dis (2007) 66(12):1679-82. doi: 10.1136/ard.2006.064626

7. Ford SL, Polkinghorne KR, Longano A, Dowling J, Dayan S, Kerr PG, et al. Histopathologic and Clinical Predictors of Kidney Outcomes in ANCAAssociated Vasculitis. Am J Kidney Dis (2014) 63(2):227-35. doi: 10.1053/ j.ajkd.2013.08.025

8. Odobasic D, Kitching AR, Holdsworth SR. Neutrophil-Mediated Regulation of Innate and Adaptive Immunity: The Role of Myeloperoxidase. J Immunol Res (2016) 2016:2349817. doi: 10.1155/2016/2349817

9. Coughlan AM, Freeley SJ, Robson MG. Animal Models of Anti-Neutrophil Cytoplasmic Antibody-Associated Vasculitis. Clin Exp Immunol (2012) 169 (3):229-37. doi: 10.1111/j.1365-2249.2012.04616.x

10. Cunningham M, Huang $X$, Dowling J, Tipping PG, Holdsworth SR. Prominence of Cell-Mediated Immunity Effectors in "Pauci-Immune" Glomerulonephritis. J Am Soc Nephrol (1999) 10(3):499-506. doi: 10.1681/ASN.V103499

11. Gan PY, Holdsworth SR, Kitching AR, Ooi JD. Myeloperoxidase (MPO)Specific CD4+ T Cells Contribute to MPO-Anti-Neutrophil Cytoplasmic Antibody (ANCA) Associated Glomerulonephritis. Cell Immunol (2013) 282 (1):21-7. doi: 10.1016/j.cellimm.2013.04.007

12. Kettritz R. How Anti-Neutrophil Cytoplasmic Autoantibodies Activate Neutrophils. Clin Exp Immunol (2012) 169(3):220-8. doi: 10.1111/j.13652249.2012.04615.x

13. Ruth A, Kitching A, Kwan R, Odobasic D, Ooi J, Timoshanko J, et al. AntiNeutrophil Cytoplasmic Antibodies and Effector CD4+ Cells Play major adverse and non-antigen-specific effects which currently occur with the existing treatments.

\section{AUTHOR CONTRIBUTIONS}

DO searched the literature and wrote the manuscript. SH reviewed and edited the paper. All authors contributed to the article and approved the submitted version.

\section{FUNDING}

The funds for these studies were used from grants provided by the National Health and Medical Research Council of Australia (NHMRC) and the Australian Government Department of Health [Medical Research Future Fund [MRFF)].

Nonredundant Roles in Anti-Myeloperoxidase Crescentic Glomerulonephritis. J Am Soc Nephrol (2006) 17:1940-9. doi: 10.1681/ ASN.2006020108

14. Xiao H, Heeringa P, Hu P, Liu Z, Zhao M, Aratani Y, et al. Antineutrophil Cytoplasmic Autoantibodies Specific for Myeloperoxidase Cause Glomerulonephritis and Vasculitis in Mice. J Clin Invest (2002) 110 (7):955-63. doi: 10.1172/JCI0215918

15. Harper L, Radford D, Plant T, Drayson M, Adu D, Savage CO. IgG From Myeloperoxidase-Antineutrophil Cytoplasmic Antibody-Positive Patients Stimulates Greater Activation of Primed Neutrophils Than IgG From Proteinase 3-Antineutrophil Cytosplasmic Antibody-Positive Patients. Arthritis Rheum (2001) 44(4):921-30. doi: 10.1002/1529-0131(200104) 44:4<921::AID-ANR149>3.0.CO;2-4

16. Ooi JD, Chang J, Hickey MJ, Borza DB, Fugger L, Holdsworth SR, et al. The Immunodominant Myeloperoxidase T-Cell Epitope Induces Local CellMediated Injury in Antimyeloperoxidase Glomerulonephritis. Proc Natl Acad Sci USA (2012) 109(39):E2615-24. doi: 10.1073/pnas.1210147109

17. Shochet L, Holdsworth S, Kitching AR. Animal Models of ANCA Associated Vasculitis. Front Immunol (2020) 11:525. doi: 10.3389/fimmu.2020.00525

18. Gan PY, Summers SA, Ooi JD, O'Sullivan KM, Tan DS, Muljadi RC, et al Mast Cells Contribute to Peripheral Tolerance and Attenuate Autoimmune Vasculitis. J Am Soc Nephrol (2012) 23(12):1955-66. doi: 10.1681/ ASN.2012060572

19. Gan PY, Steinmetz OM, Tan DS, O'Sullivan KM, Ooi JD, Iwakura Y, et al. Th17 Cells Promote Autoimmune Anti-Myeloperoxidase Glomerulonephritis. J Am Soc Nephrol (2010) 21(6):925-31. doi: 10.1681/ ASN.2009070763

20. Roth AJ, Ooi JD, Hess JUJ, van Timmeren MM, Berg EA, Poulton CE, et al. Epitope Specificity Determines Pathogenicity and Detectability in ANCAAssociated Vasculitis. J Clin Invest (2013) 123(4):1773-83. doi: 10.1172/ JCI65292

21. Ooi JD, Jiang JH, Eggenhuizen PJ, Chua LL, van Timmeren M, Loh KL, et al. A Plasmid-Encoded Peptide From Staphylococcus Aureus Induces AntiMyeloperoxidase Nephritogenic Autoimmunity. Nat Commun (2019) 10 (1):3392. doi: 10.1038/s41467-019-11255-0

22. O’Sullivan K, Lo C, Summers S, Elgass K, McMillan P, Longano A, et al. Renal Participation of Myeloperoxidase in Antineutrophil Cytoplasmic Antibody (ANCA)-Associated Glomerulonephritis. Kidney Int (2015) 88 (5):1030-46. doi: 10.1038/ki.2015.202

23. Chang J, Eggenhuizen P, O'Sullivan KM, Alikhan MA, Holdsworth SR, Ooi JD, et al. CD8+ T Cells Effect Glomerular Injury in Experimental AntiMyeloperoxidase GN. J Am Soc Nephrol (2017) 28(1):47-55. doi: 10.1681/ ASN.2015121356

24. Rousselle A, Kettritz R, Schreiber A. Monocytes Promote Crescent Formation in Anti-Myeloperoxidase Antibody-Induced 
Glomerulonephritis. Am J Pathol (2017) 187(9):1908-15. doi: 10.1016/ j.ajpath.2017.05.003

25. Nogueira E, Hamour S, Sawant D, Henderson S, Mansfield N, Chavele KM, et al. Serum IL-17 and IL-23 Levels and Autoantigen-Specific Th17 Cells are Elevated in Patients With ANCA-Associated Vasculitis. Nephrol Dial Transplant (2010) 25(7):2209-17. doi: 10.1093/ndt/gfp783

26. Summers SA, Steinmetz OM, Gan PY, Ooi JD, Odobasic D, Kitching AR, et al. Toll-Like Receptor 2 Induces Th17 Myeloperoxidase Autoimmunity While Toll-Like Receptor 9 Drives Th1 Autoimmunity in Murine Vasculitis. Arthritis Rheum (2011) 63(4):1124-35. doi: 10.1002/art.30208

27. Yoshida M, Iwahori T, Nakabayashi I, Akashi M, Watanabe T, Yoshikawa N. In Vitro Production of Myeloperoxidase Anti-Neutrophil Cytoplasmic Antibody and Establishment of Th1-Type T Cell Lines From Peripheral Blood Lymphocytes of Patients. Clin Exp Rheumatol (2005) 23(2):227-30.

28. Free ME, Bunch DO, McGregor JA, Jones BE, Berg EA, Hogan SL, et al. Patients With Antineutrophil Cytoplasmic Antibody-Associated Vasculitis Have Defective Treg Cell Function Exacerbated by the Presence of a Suppression-Resistant Effector Cell Population. Arthritis Rheum (2013) 65 (7):1922-33. doi: 10.1002/art.37959

29. Tan DS, Gan PY, O'Sullivan KM, Hammett MV, Summers SA, Ooi JD, et al. Thymic Deletion and Regulatory T Cells Prevent Antimyeloperoxidase GN. J Am Soc Nephrol (2013) 24(4):573-85. doi: 10.1681/ASN.2012090898

30. von Borstel A, Sanders JS, Rutgers A, Stegeman CA, Heeringa P, Abdulahad $\mathrm{WH}$, et al. Cellular Immune Regulation in the Pathogenesis of ANCAAssociated Vasculitides. Autoimmun Rev (2018) 17(4):413-21. doi: 10.1016/ j.autrev.2017.12.002

31. Flossmann O, Berden A, de Groot K, Hagen C, Harper L, Heijl C, et al. LongTerm Patient Survival in ANCA-Associated Vasculitis. Ann Rheum Dis (2011) 70(3):488-94. doi: 10.1136/ard.2010.137778

32. King C, Harper L. Avoidance of Harm From Treatment for ANCAAssociated Vasculitis. Curr Treatm Opt Rheumatol (2017) 3(4):230-43. doi: 10.1007/s40674-017-0082-y

33. Robson JC, Dawson J, Cronholm PF, Ashdown S, Easley E, Kellom KS, et al. Patient Perceptions of Glucocorticoids in Anti-Neutrophil Cytoplasmic Antibody-Associated Vasculitis. Rheumatol Int (2018) 38(4):675-82. doi: 10.1007/s00296-017-3855-6

34. Jayne DRW, Bruchfeld AN, Harper L, Schaier M, Venning MC, Hamilton P, et al. Randomized Trial of C5a Receptor Inhibitor Avacopan in ANCAAssociated Vasculitis. J Am Soc Nephrol (2017) 28(9):2756-67. doi: 10.1681/ ASN.2016111179

35. Jayne DRW, Merkel PA, Schall TJ, Bekker P, Group AS. Avacopan for the Treatment of ANCA-Associated Vasculitis. N Engl J Med (2021) 384(7):599609. doi: $10.1056 / \mathrm{NEJMoa} 2023386$

36. Jones RB, Furuta S, Tervaert JW, Hauser T, Luqmani R, Morgan MD, et al. Rituximab Versus Cyclophosphamide in ANCA-Associated Renal Vasculitis: 2-Year Results of a Randomised Trial. Ann Rheum Dis (2015) 74(6):1178-82. doi: 10.1136/annrheumdis-2014-206404

37. Hasegawa $\mathrm{H}$, Matsumoto T. Mechanisms of Tolerance Induction by Dendritic Cells In Vivo. Front Immunol (2018) 9:350. doi: 10.3389/fimmu.2018.00350

38. Ohnmacht C, Pullner A, King SB, Drexler I, Meier S, Brocker T, et al. Constitutive Ablation of Dendritic Cells Breaks Self-Tolerance of CD4 T Cells and Results in Spontaneous Fatal Autoimmunity. J Exp Med (2009) 206 (3):549-59. doi: $10.1084 /$ jem.20082394

39. Engman C, Garciafigueroa Y, Phillips BE, Trucco M, Giannoukakis N, et al. Co-Stimulation-Impaired Bone Marrow-Derived Dendritic Cells Prevent Dextran Sodium Sulfate-Induced Colitis in Mice. Front Immunol (2018) 9:894. doi: 10.3389/fimmu.2018.00894

40. Steinbrink K, Wolfl M, Jonuleit H, Knop J, Enk AH. Induction of Tolerance by IL-10-Treated Dendritic Cells. J Immunol (1997) 159(10):4772-80.

41. Martin E, Capini C, Duggan E, Lutzky VP, Stumbles P, Pettit AR, et al. Antigen-Specific Suppression of Established Arthritis in Mice by Dendritic Cells Deficient in NF-kappaB. Arthritis Rheum (2007) 56(7):2255-66. doi: 10.1002/art.22655

42. Stoop JN, Harry RA, von Delwig A, Isaacs JD, Robinson JH, Hilkens CM, et al. Therapeutic Effect of Tolerogenic Dendritic Cells in Established Collagen-Induced Arthritis is Associated With a Reduction in Th17 Responses. Arthritis Rheum (2010) 62(12):3656-65. doi: 10.1002/art.27756
43. Ferreira GB, Gysemans CA, Demengeot J, da Cunha JP, Vanherwegen AS, Overbergh L, et al. 1,25-Dihydroxyvitamin D3 Promotes Tolerogenic Dendritic Cells With Functional Migratory Properties in NOD Mice. J Immunol (2014) 192(9):4210-20. doi: 10.4049/jimmunol.1302350

44. Martin E, O'Sullivan B, Low P, Thomas R. Antigen-Specific Suppression of a Primed Immune Response by Dendritic Cells Mediated by Regulatory $\mathrm{T}$ Cells Secreting Interleukin-10. Immunity (2003) 18(1):155-67. doi: 10.1016/S1074-7613(02)00503-4

45. Tai N, Yasuda H, Xiang Y, Zhang L, Rodriguez-Pinto D, Yokono K, et al. IL10-Conditioned Dendritic Cells Prevent Autoimmune Diabetes in NOD and Humanized HLA-DQ8/RIP-B7.1 Mice. Clin Immunol (2011) 139(3):336-49. doi: 10.1016/j.clim.2011.03.003

46. Thomas DC, Wong FS, Zaccone P, Green EA, Wallberg M. Protection of Islet Grafts Through Transforming Growth Factor-Beta-Induced Tolerogenic Dendritic Cells. Diabetes (2013) 62(9):3132-42. doi: 10.2337/ $\mathrm{db} 12-1740$

47. Qian C, Qian L, Yu Y, An H, Guo Z, Han Y, et al. Fas Signal Promotes the Immunosuppressive Function of Regulatory Dendritic Cells via the ERK/ beta-Catenin Pathway. J Biol Chem (2013) 288(39):27825-35. doi: 10.1074/ jbc.M112.425751

48. Iruretagoyena MI, Sepulveda SE, Lezana JP, Hermoso M, Bronfman M, Gutierrez MA, et al. Inhibition of Nuclear Factor-Kappa B Enhances the Capacity of Immature Dendritic Cells to Induce Antigen-Specific Tolerance in Experimental Autoimmune Encephalomyelitis. J Pharmacol Exp Ther (2006) 318(1):59-67. doi: 10.1124/jpet.106.103259

49. Ma L, Qian S, Liang X, Wang L, Woodward JE, Giannoukakis N, et al. Prevention of Diabetes in NOD Mice by Administration of Dendritic Cells Deficient in Nuclear Transcription factor-kappaB Activity. Diabetes (2003) 52(8):1976-85. doi: 10.2337/diabetes.52.8.1976

50. Suzuki M, Zheng X, Zhang X, Zhang ZX, Ichim TE, Sun H, et al. A Novel Allergen-Specific Therapy for Allergy Using CD40-Silenced Dendritic Cells. J Allergy Clin Immunol (2010) 125(3):737-43, 743 e1-743 e6. doi: 10.1016/ j.jaci.2009.11.042

51. Ezzelarab MB, Raich-Regue D, Lu L, Zahorchak AF, Perez-Gutierrez A, Humar A, et al. Renal Allograft Survival in Nonhuman Primates Infused With Donor Antigen-Pulsed Autologous Regulatory Dendritic Cells. Am J Transplant (2017) 17(6):1476-89. doi: 10.1111/ajt.14182

52. Machen J, Harnaha J, Lakomy R, Styche A, Trucco M, Giannoukakis N. Antisense Oligonucleotides Down-Regulating Costimulation Confer Diabetes-Preventive Properties to Nonobese Diabetic Mouse Dendritic Cells. J Immunol (2004) 173(7):4331-41. doi: 10.4049/jimmunol.173.7.4331

53. Benham H, Nel HJ, Law SC, Mehdi AM, Street S, Ramnoruth N, et al. Citrullinated Peptide Dendritic Cell Immunotherapy in HLA Risk Genotype-Positive Rheumatoid Arthritis Patients. Sci Transl Med (2015) 7 (290):290ra87. doi: 10.1126/scitranslmed.aaa9301

54. Giannoukakis N, Phillips B, Finegold D, Harnaha J, Trucco M. Phase I (Safety) Study of Autologous Tolerogenic Dendritic Cells in Type 1 Diabetic Patients. Diabetes Care (2011) 34(9):2026-32. doi: 10.2337/dc11-0472

55. Jauregui-Amezaga A, Cabezon R, Ramirez-Morros A, Espana C, Rimola J, Bru C, et al. Intraperitoneal Administration of Autologous Tolerogenic Dendritic Cells for Refractory Crohn's Disease: A Phase I Study. J Crohns Colitis (2015) 9(12):1071-8. doi: 10.1093/ecco-jcc/jjv144

56. Bell GM, Anderson AE, Diboll J, Reece R, Eltherington O, Harry RA, et al. Autologous Tolerogenic Dendritic Cells for Rheumatoid and Inflammatory Arthritis. Ann Rheum Dis (2017) 76(1):227-34. doi: 10.1136/annrheumdis2015-208456

57. Zubizarreta I, Florez-Grau G, Vila G, Cabezon R, Espana C, Andorra M, et al. Immune Tolerance in Multiple Sclerosis and Neuromyelitis Optica With Peptide-Loaded Tolerogenic Dendritic Cells in a Phase 1b Trial. Proc Natl Acad Sci USA (2019) 116(17):8463-70. doi: 10.1073/pnas.1820039116

58. Odobasic D, Oudin V, Ito K, Gan PY, Kitching AR, Holdsworth SR Tolerogenic Dendritic Cells Attenuate Experimental Autoimmune Antimyeloperoxidase Glomerulonephritis. J Am Soc Nephrol (2019) 30 (11):2140-57. doi: 10.1681/ASN.2019030236

59. Wan YY, Flavell RA. Regulatory T-Cell Functions are Subverted and Converted Owing to Attenuated Foxp3 Expression. Nature (2007) 445 (7129):766-70. doi: 10.1038/nature05479 
60. Rimbert M, Hamidou M, Braudeau C, Puechal X, Teixeira L, Caillon H, et al. Decreased Numbers of Blood Dendritic Cells and Defective Function of Regulatory T Cells in Antineutrophil Cytoplasmic Antibody-Associated Vasculitis. PLoS One (2011) 6(4):e18734. doi: 10.1371/journal.pone.0018734

61. Veerapathran A, Pidala J, Beato F, Betts B, Kim J, Turner JG, et al. Human Regulatory T Cells Against Minor Histocompatibility Antigens: Ex Vivo Expansion for Prevention of Graft-Versus-Host Disease. Blood (2013) 122 (13):2251-61. doi: 10.1182/blood-2013-03-492397

62. Josefowicz SZ, Lu LF, Rudensky AY. Regulatory T Cells: Mechanisms of Differentiation and Function. Annu Rev Immunol (2012) 30:531-64. doi: 10.1146/annurev.immunol.25.022106.141623

63. Kleijwegt FS, Laban S, Duinkerken G, Joosten AM, Koeleman BP, Nikolic T, et al. Transfer of Regulatory Properties From Tolerogenic to Proinflammatory Dendritic Cells via Induced Autoreactive Regulatory T Cells. J Immunol (2011) 187(12):6357-64. doi: 10.4049/jimmunol.1101638

64. Eggenhuizen PJ, Ng BH, Ooi JD. Treg Enhancing Therapies to Treat Autoimmune Diseases. Int J Mol Sci (2020) 21(19):7015. doi: 10.3390/ ijms 21197015

65. Chen X, Baumel M, Mannel DN, Howard OM, Oppenheim JJ, et al. Interaction of TNF With TNF Receptor Type 2 Promotes Expansion and Function of Mouse CD4+CD25+ T Regulatory Cells. J Immunol (2007) 179 (1):154-61. doi: 10.4049/jimmunol.179.1.154

66. Read S, Malmstrom V, Powrie F. Cytotoxic T Lymphocyte-Associated Antigen 4 Plays an Essential Role in the Function of CD25(+)CD4(+) Regulatory Cells That Control Intestinal Inflammation. J Exp Med (2000) 192(2):295-302. doi: 10.1084/jem.192.2.295

67. Joffre O, Santolaria T, Calise D, Al Saati T, Hudrisier D, Romagnoli P, et al. Prevention of Acute and Chronic Allograft Rejection With CD4+CD25 +Foxp3+ Regulatory T Lymphocytes. Nat Med (2008) 14(1):88-92. doi: $10.1038 / \mathrm{nm} 1688$

68. Tang Q, Henriksen KJ, Bi M, Finger EB, Szot G, Ye J, et al. In VitroExpanded Antigen-Specific Regulatory T Cells Suppress Autoimmune Diabetes. J Exp Med (2004) 199(11):1455-65. doi: 10.1084/jem.20040139

69. Fransson M, Piras E, Burman J, Nilsson B, Essand M, Lu B, et al. CAR/ FoxP3-Engineered T Regulatory Cells Target the CNS and Suppress EAE Upon Intranasal Delivery. J Neuroinflamm (2012) 9:112. doi: 10.1186/17422094-9-112

70. Bluestone JA, Buckner JH, Fitch M, Gitelman SE, Gupta S, Hellerstein MK, et al. Type 1 Diabetes Immunotherapy Using Polyclonal Regulatory T Cells. Sci Transl Med (2015) 7(315):315ra189. doi: 10.1126/scitranslmed.aad4134

71. Marek-Trzonkowska N, Mysliwiec M, Dobyszuk A, Grabowska M, Derkowska I, Juscinska J, et al. Therapy of Type 1 Diabetes With CD4(+) CD25(high)CD127-Regulatory T Cells Prolongs Survival of Pancreatic Islets - Results of One Year Follow-Up. Clin Immunol (2014) 153(1):23-30. doi: 10.1016/j.clim.2014.03.016

72. Ooi JD, Petersen J, Tan YH, Huynh M, Willett ZJ, Ramarathinam SH, et al. Dominant Protection From HLA-Linked Autoimmunity by AntigenSpecific Regulatory T Cells. Nature (2017) 545(7653):243-7. doi: 10.1038/ nature22329

73. Hoffmann P, Boeld TJ, Eder R, Huehn J, Floess S, Wieczorek G, et al. Loss of FOXP3 Expression in Natural Human CD4+CD25+ Regulatory T Cells Upon Repetitive In Vitro Stimulation. Eur J Immunol (2009) 39(4):1088-97. doi: 10.1002/eji.200838904

74. Free ME, Stember KG, Hess JJ, McInnis EA, Lardinois O, Hogan SL, et al. Restricted Myeloperoxidase Epitopes Drive the Adaptive Immune Response in MPO-ANCA Vasculitis. J Autoimmun (2020) 106:102306. doi: 10.1016/ j.jaut.2019.102306

75. Khalilova IS, Dickerhof N, Mocatta TJ, Bhagra CJ, McClean DR, Obinger C, et al. A Myeloperoxidase Precursor, Pro-Myeloperoxidase, is Present in Human Plasma and Elevated in Cardiovascular Disease Patients. PLoS One (2018) 13(3):e0192952. doi: 10.1371/journal.pone.0192952

76. Yamazaki S, Inaba K, Tarbell KV, Steinman RM. Dendritic Cells Expand Antigen-Specific Foxp3+ CD25+ CD4+ Regulatory T Cells Including Suppressors of Alloreactivity. Immunol Rev (2006) 212:314-29. doi: 10.1111/j.0105-2896.2006.00422.x

77. Pothoven KL, Kheradmand T, Yang Q, Houlihan JL, Zhang H, Degutes M, et al. Rapamycin-Conditioned Donor Dendritic Cells Differentiate CD4CD25Foxp3 T Cells In Vitro With TGF-Beta1 for Islet
Transplantation. Am J Transplant (2010) 10(8):1774-84. doi: 10.1111/ j.1600-6143.2010.03199.x

78. Yang J, Liu L, Yang Y, Kong N, Jiang X, Sun J, et al. Adoptive Cell Therapy of Induced Regulatory T Cells Expanded by Tolerogenic Dendritic Cells on Murine Autoimmune Arthritis. J Immunol Res (2017) 2017:7573154. doi: $10.1155 / 2017 / 7573154$

79. Muzes G, Sipos F. Issues and Opportunities of Stem Cell Therapy in Autoimmune Diseases. World J Stem Cells (2019) 11(4):212-21. doi: 10.4252/wjsc.v11.i4.212

80. Miki T, Lehmann T, Cai H, Stolz DB, Strom SC. Stem Cell Characteristics of Amniotic Epithelial Cells. Stem Cells (2005) 23(10):1549-59. doi: 10.1634/ stemcells.2004-0357

81. Broughton BR, Lim R, Arumugam TV, Drummond GR, Wallace EM, Sobey CG. Post-Stroke Inflammation and the Potential Efficacy of Novel Stem Cell Therapies: Focus on Amnion Epithelial Cells. Front Cell Neurosci (2012) 6:66. doi: 10.3389/fncel.2012.00066

82. Murphy S, Rosli S, Acharya R, Mathias L, Lim R, Wallace E, et al. Amnion Epithelial Cell Isolation and Characterization for Clinical Use. Curr Protoc Stem Cell Biol (2010), Unit 1E 6. Chapter 1. doi: 10.1002/9780470151808. sc01e06s13

83. Moodley Y, Ilancheran S, Samuel C, Vaghjiani V, Atienza D, Williams ED, et al. Human Amnion Epithelial Cell Transplantation Abrogates Lung Fibrosis and Augments Repair. Am J Respir Crit Care Med (2010) 182 (5):643-51. doi: 10.1164/rccm.201001-0014OC

84. Parmar DN, Alizadeh H, Awwad ST, Li H, Neelam S, Bowman RW, et al. Ocular Surface Restoration Using non-Surgical Transplantation of TissueCultured Human Amniotic Epithelial Cells. Am J Ophthalmol (2006) 141 (2):299-307. doi: 10.1016/j.ajo.2005.09.008

85. Togel F, Hu Z, Weiss K, Isaac J, Lange C, Westenfelder C. Administered Mesenchymal Stem Cells Protect Against Ischemic Acute Renal Failure Through Differentiation-Independent Mechanisms. Am J Physiol Renal Physiol (2005) 289(1):F31-42. doi: 10.1152/ajprenal.00007.2005

86. Wang Y, Chen X, Cao W, Shi Y. Plasticity of Mesenchymal Stem Cells in Immunomodulation: Pathological and Therapeutic Implications. Nat Immunol (2014) 15(11):1009-16. doi: 10.1038/ni.3002

87. Li H, Niederkorn JY, Neelam S, Mayhew E, Word RA, McCulley JP, et al. Immunosuppressive Factors Secreted by Human Amniotic Epithelial Cells. Invest Ophthalmol Vis Sci (2005) 46(3):900-7. doi: 10.1167/iovs.04-0495

88. Tan JL, Lau SN, Leaw B, Nguyen HPT, Salamonsen LA, Saad MI, et al. Amnion Epithelial Cell-Derived Exosomes Restrict Lung Injury and Enhance Endogenous Lung Repair. Stem Cells Transl Med (2018) 7 (2):180-96. doi: 10.1002/sctm.17-0185

89. Liu YH, Vaghjiani V, Tee JY, To K, Cui P, Oh DY, et al. Amniotic Epithelial Cells From the Human Placenta Potently Suppress a Mouse Model of Multiple Sclerosis. PLoS One (2012) 7(4):e35758. doi: 10.1371/ journal.pone. 0035758

90. Manuelpillai U, Tchongue J, Lourensz D, Vaghjiani V, Samuel CS, Liu A, et al. Transplantation of Human Amnion Epithelial Cells Reduces Hepatic Fibrosis in Immunocompetent CCl(4)-Treated Mice. Cell Transplant (2010) 19(9):1157-68. doi: 10.3727/096368910X504496

91. Tan JL, Chan ST, Lo CY, Deane JA, McDonald CA, Bernard CC, et al. Amnion Cell Mediated Immune Modulation Following Bleomycin Challenge: Controlling the Regulatory T Cell Response. Stem Cell Res Ther (2015) 6(1):8. doi: 10.1186/scrt542

92. Tan JL, Chan ST, Wallace EM, Lim R. Human Amnion Epithelial Cells Mediate Lung Repair by Directly Modulating Macrophage Recruitment and Polarization. Cell Transplant (2014) 23(3):319-28. doi: 10.3727/ 096368912X661409

93. Li J, Qiu C, Zhang Z, Yuan W, Ge Z, Tan B, et al. Subretinal Transplantation of Human Amniotic Epithelial Cells in the Treatment of Autoimmune Uveitis in Rats. Cell Transplant (2018) 27(10):1504-14. doi: 10.1177/ 0963689718796196

94. Tan B, Yuan W, Li J, Yang P, Ge Z, Liu J, et al. Therapeutic Effect of Human Amniotic Epithelial Cells in Murine Models of Hashimoto's Thyroiditis and Systemic Lupus Erythematosus. Cytotherapy (2018) 20(10):1247-58. doi: 10.1016/j.jcyt.2018.04.001

95. Evans MA, Lim R, Kim HA, Chu HX, Gardiner-Mann CV, Taylor KWE, et al. Acute or Delayed Systemic Administration of Human Amnion 
Epithelial Cells Improves Outcomes in Experimental Stroke. Stroke (2018) 49(3):700-9. doi: 10.1161/STROKEAHA.117.019136

96. Motedayyen H, Zarnani AH, Tajik N, Ghotloo S, Rezaei A. Immunomodulatory Effects of Human Amniotic Epithelial Cells on Naive CD4(+) T Cells From Women With Unexplained Recurrent Spontaneous Abortion. Placenta (2018) 71:31-40. doi: 10.1016/j.placenta.2018.06.008

97. Wolbank S, Peterbauer A, Fahrner M, Hennerbichler S, Griensven M, Stadler G, et al. Dose-Dependent Immunomodulatory Effect of Human Stem Cells From Amniotic Membrane: A Comparison With Human Mesenchymal Stem Cells From Adipose Tissue. Tissue Eng (2007) 13 (6):1173-83. doi: 10.1089/ten.2006.0313

98. Lim R, Chan ST, Tan JL, Mockler JC, Murphy SV, Wallace EM. Preterm Human Amnion Epithelial Cells Have Limited Reparative Potential. Placenta (2013) 34(6):486-92. doi: 10.1016/j.placenta.2013.03.010

99. Baudhuin J, Migraine J, Faivre V, Loumagne L, Lukaszewicz AC, Payen D, et al. Exocytosis Acts as a Modulator of the ILT4-Mediated Inhibition of Neutrophil Functions. Proc Natl Acad Sci USA (2013) 110(44):17957-62. doi: $10.1073 /$ pnas. 1221535110

100. Rizzo R, Bortolotti D, Bolzani S, Fainardi E. HLA-G Molecules in Autoimmune Diseases and Infections. Front Immunol (2014) 5:592. doi: 10.3389/fimmu.2014.00592

101. Alhomrani M, Correia J, Zavou M, Leaw B, Kuk N, Xu R, et al. The Human Amnion Epithelial Cell Secretome Decreases Hepatic Fibrosis in Mice With Chronic Liver Fibrosis. Front Pharmacol (2017) 8:748. doi: 10.3389/ fphar.2017.00748

102. Zhang B, Yin Y, Lai RC, Lim SK. Immunotherapeutic Potential of Extracellular Vesicles. Front Immunol (2014) 5:518. doi: 10.3389/fimmu. 2014.00518

103. Lee C, Mitsialis SA, Aslam M, Vitali SH, Vergadi E, Konstantinou G, et al. Exosomes Mediate the Cytoprotective Action of Mesenchymal Stromal Cells on Hypoxia-Induced Pulmonary Hypertension. Circulation (2012) 126 (22):2601-11. doi: 10.1161/CIRCULATIONAHA.112.114173

104. Gatti S, Bruno S, Deregibus MC, Sordi A, Cantaluppi V, Tetta C, et al. Microvesicles Derived From Human Adult Mesenchymal Stem Cells Protect Against Ischaemia-Reperfusion-Induced Acute and Chronic Kidney Injury. Nephrol Dial Transplant (2011) 26(5):1474-83. doi: 10.1093/ndt/ gfr015

105. Nemr W, Bashandy M, Araby E, Khamiss O. Molecular Displaying of Differential Immunoresponse to Various Infections of Amniotic Epithelia. Am J Reprod Immunol (2017) 77(6). doi: 10.1111/aji.12662

106. Uchide N, Ohyama K, Yuan B, Sano T, Bessho T, Yamakawa T, et al. Differential mRNA Expression of Inflammatory Cytokines in Cultured Human Fetal Membrane Cells Responding to Influenza Virus Infection. Biol Pharm Bull (2002) 25(2):239-43. doi: 10.1248/bpb.25.239

107. Niknejad H, Khayat-Khoei M, Peirovi H, Abolghasemi H. Human Amniotic Epithelial Cells Induce Apoptosis of Cancer Cells: A New Anti-Tumor
Therapeutic Strategy. Cytotherapy (2014) 16(1):33-40. doi: 10.1016/ j.jcyt.2013.07.005

108. Tabatabaei M, Mosaffa N, Ghods R, Nikoo S, Kazemnejad S, Khanmohammadi M, et al. Vaccination With Human Amniotic Epithelial Cells Confer Effective Protection in a Murine Model of Colon Adenocarcinoma. Int J Cancer (2018) 142(7):1453-66. doi: 10.1002/ijc.31159

109. Fang CH, Jin J, Joe JH, Song YS, So BI, Lim SM, et al. In Vivo Differentiation of Human Amniotic Epithelial Cells Into Cardiomyocyte-Like Cells and Cell Transplantation Effect on Myocardial Infarction in Rats: Comparison With Cord Blood and Adipose Tissue-Derived Mesenchymal Stem Cells. Cell Transplant (2012) 21(8):1687-96. doi: 10.3727/096368912X653039

110. Jirsova K, Jones GLA. Amniotic Membrane in Ophthalmology: Properties, Preparation, Storage and Indications for Grafting-a Review. Cell Tissue Bank (2017) 18(2):193-204. doi: 10.1007/s10561-017-9618-5

111. Lim R, Malhotra A, Tan J, Chan ST, Lau S, Zhu D, et al. First-In-Human Administration of Allogeneic Amnion Cells in Premature Infants With Bronchopulmonary Dysplasia: A Safety Study. Stem Cells Transl Med (2018) 7(9):628-35. doi: 10.1002/sctm.18-0079

112. Malhotra A, Lim R, Mockler JC, Wallace EM, et al. Two-Year Outcomes of Infants Enrolled in the First-in-Human Study of Amnion Cells for Bronchopulmonary Dysplasia. Stem Cells Transl Med (2020) 9(3):289-94. doi: $10.1002 / \mathrm{sctm} .19-0251$

113. Lim R, Hodge A, Moore G, Wallace EM, Sievert W. A Pilot Study Evaluating the Safety of Intravenously Administered Human Amnion Epithelial Cells for the Treatment of Hepatic Fibrosis. Front Pharmacol (2017) 8:549. doi: 10.3389/fphar.2017.00549

114. Phan TG, Ma H, Lim R, Sobey CG, Wallace EM, et al. Phase 1 Trial of Amnion Cell Therapy for Ischemic Stroke. Front Neurol (2018) 9:198. doi: 10.3389/fneur.2018.00198

Conflict of Interest: The authors declare that the research was conducted in the absence of any commercial or financial relationships that could be construed as a potential conflict of interest.

Publisher's Note: All claims expressed in this article are solely those of the authors and do not necessarily represent those of their affiliated organizations, or those of the publisher, the editors and the reviewers. Any product that may be evaluated in this article, or claim that may be made by its manufacturer, is not guaranteed or endorsed by the publisher.

Copyright $\odot 2021$ Odobasic and Holdsworth. This is an open-access article distributed under the terms of the Creative Commons Attribution License (CC BY). The use, distribution or reproduction in other forums is permitted, provided the original author(s) and the copyright owner(s) are credited and that the original publication in this journal is cited, in accordance with accepted academic practice. No use, distribution or reproduction is permitted which does not comply with these terms. 